\title{
Asociación entre los rasgos de personalidad y la ocurrencia de accidentes de trabajo de riesgo biológico del personal de enfermería en el Hospital Universitario de Santander (HUS): Estudio de casos y controles
}

Association between personality traits and the occurrence of work accidents involving biological hazards among nursing staff at Hospital Universitario de Santander (HUS): Case-control study

Associação entre traços de personalidade e ocorrência de acidentes de trabalho com risco biológico em profissionais de enfermagem do Hospital Universitário de Santander (HUS): Estudo caso-controle

Adriana Arenas-Sánchez, Enf., Mg., Esp. ${ }^{1}$ (D), Alexander Pinzón-Amaya, MD., Mg., Esp. ${ }^{2}$ (D)

1. Enfermera, Magister en Epidemiología, Especialista en Administración de Servicios de Salud, Especialista en Salud Ocupacional. Universidad Industrial de Santander.

2. Médico, Especialista en Psiquiatría, Magister en Epidemiología Clínica. Universidad Industrial de Santander.

Correspondencia. Adriana Arenas Sánchez. Carrera 2 No. 200-105 apto 903 Firenze Platino. Email: arenasepi@gmail.com

\section{INFORMACIÓN DEL ARTÍCULO:}

Artículo recibido: 31 de agosto de 2020

Artículo aceptado: 25 de junio de 2021

DOI: https://doi.org/10.29375/01237047.3981

Cómo citar: Arenas-Sánchez A, Pinzón-Amaya A. Asociación entre los rasgos de personalidad y la ocurrencia de accidentes de trabajo de riesgo biológico del personal de enfermería en el Hospital Universitario de Santander (HUS): Estudio de casos y controles. MedUNAB. 2021; 24(2):220-232 doi: https://doi.org/10.29375/01237047.3981

\section{RESUMEN}

Introducción. Los accidentes de trabajo por exposición al riesgo biológico (ATBIO) son causas potenciales de enfermedades infecciosas relacionadas con alta morbilidad y mortalidad. La identificación de las causas que generan dicha accidentalidad permitirá establecer estrategias que controlen y disminuyan la siniestralidad laboral. El objetivo del presente manuscrito fue establecer la asociación entre los rasgos de personalidad, principalmente el rasgo de personalidad de despreocupación y la 


\section{Introducción}

La definición del evento "accidente de trabajo" (AT) en la legislación colombiana es: "todo suceso repentino que sobrevenga por causa o con ocasión del trabajo y que produzca en el trabajador una lesión orgánica, una perturbación funcional, una invalidezo la muerte. También, es aquel que se produce durante la ejecución de órdenes del empleador, o durante la ejecución de una labor bajo su autoridad, aún fuera del lugar y horas de trabajo" (1). Las estrategias de control para disminuir la siniestralidad laboral dependen en gran medida de la identificación de las causas que generan dicha accidentalidad, por esta razón, determinar qué factores influencian la ocurrencia de accidentes laborales permitirá implementar medidas de prevención de AT (2).

Los profesionales del área de la salud, particularmente el personal médico y de enfermería, desarrollan sus actividades laborales inmersos en un ambiente de alta exposición a diferentes tipos de riesgos, tales como riesgos biológicos, físicos, químicos, psicológicos, ergonómicos y mecánicos (3). En general, la exposición accidental ocupacional de riesgo biológico resulta ser la más frecuente para el personal de enfermería $(2,3)$. La Organización Mundial de la Salud (OMS) estima que cada año más de 3 millones de trabajadores de la salud están expuestos a sufrir un accidente de trabajo de riesgo biológico (ATBIO) por causa de alguno de los siguientes tres virus, virus de inmunodeficiencia humana (VIH), Hepatitis B y Hepatitis C (4). En países en los cuales se reporta una prevalencia baja $(<1 \%)$ de infección por estos virus en la población general, el personal de salud desarrolla una percepción de falsa de seguridad, que contribuye a una actitud de despreocupación e incumplimiento de las medidas de seguridad y uso de implemento de protección personal (2). Otros estudios han descrito cómo la falta de conocimiento sobre la patogenicidad, el modo de transmisión y las actitudes frente al seguimiento de protocolos y normas de bioseguridad incrementan el riesgo de exposición individual que propician la presentación de accidentes laborales de riesgo biológico (5-9). El riesgo de infectarse con un patógeno durante la actividad laboral por lesiones percutáneas, o por el contacto con membranas mucosas o piel no intacta de sangre, tejidos $u$ otros fluidos corporales potencialmente contaminados (5) podría ser influenciada por las actitudes y conocimiento del personal de salud frente a los riesgos que implica trabajar expuestos a diferentes categorías de patógenos (5-9).

En la actualidad se reportan las tasas más altas de exposición laboral por riesgo biológico en la última década debido a la exposición a la enfermedad del coronavirus 2019 (COVID-19) (10). Son diversos factores los que incrementan probabilidad de contagio por patógenos, entre estos están la falta de seguimiento estricto de protocolos, normas de bioseguridad y de implementación de medidas de protección personal, principalmente en países en vías de desarrollo (11). La clasificación del SARS-CoV-2 como agente biológico del grupo 3 significa que al adquirir COVID-19 como accidente de trabajo o enfermedad profesional, esta tiene repercusiones temporales y permanentes, como por ejemplo, incapacidad, secuelas y muerte (12). Anteriormente se consideraba una mayor probabilidad de contagio por patógenos como virus de inmunodeficiencia humana (VIH), Hepatitis B y Hepatitis C $(3,7,9)$. Considerando las secuelas temporales y permanentes que están asociadas a los accidentes de trabajo de riesgo biológico se hace imprescindible implementar medidas preventivas que permitan determinar si ciertos rasgos de personalidad podrían ser más proclives a verse afectados por una mayor exposición.

La personalidad es definida como el conjunto de diferencias y características en los patrones de pensamiento, percepción y comportamiento de un individuo (13). Aunque diversas teorías han intentado generalizar los factores biológicos y ambientales que determinan la personalidad y los rasgos de personalidad, sigue siendo un desafío unificar dicho concepto para las diferentes áreas de la psicología clínica y del trabajo, o selección de personal (14). La psicología de la seguridad está orientada a identificar y estudiar las variables que hacen parte de la conducta de seguridad, como el aspecto psicológico del individuo, la interacción grupal, el sistema de organización, las relaciones sociales y el ambiente laboral, en busca de establecer diagnósticos precisos e implementar medidas que orienten a la disminución de los accidentes (15). La personalidad del trabajador influye tanto en la percepción de la realidad, como en la respuesta ante distintas circunstancias laborales porque las características propias de cada trabajador determinan la dimensión de sus reacciones y de las consecuencias de sus actos (16).

De acuerdo con la Teoría del comportamiento laboral con propósito propuesta por Barrick, Mount, and Li (2013), los rasgos de personalidad de un individuo se relacionan directamente con sus motivaciones personales y buen desempeño en el trabajo (17). Este estudio sugiere que también ciertos rasgos de personalidad influencian el desempeño laboral de manera positiva o negativa (18). En un estudio realizado con enfermeras que se expusieron a ATBIO por fluidos como sangre en seis escuelas de enfermería en Francia, se establece la relación entre la personalidad y el número de accidentes de trabajo, cuatro variables se vincularon significativa e independientemente al riesgo de tener un mayor número de ATBIO: tener un puesto fijo, tener un mayor grado de desinhibición, ser 
más susceptible al aburrimiento y tener menos experiencia en enfermería (19). En la regresión logística surgieron tres variables que se relacionaron de manera significativa e independiente con el informe de todos los ATBIO: edad más joven; haber tenido al menos una lesión percutánea (excluidas las salpicaduras); y tener menor susceptibilidad al aburrimiento (19). Este estudio concluye que el personal de enfermería continúa ignorando o desconociendo muchos factores que rodean a los ATBIO (19). Finalmente, ciertos rasgos de personalidad, como un alto nivel de desinhibición y susceptibilidad al aburrimiento, parecen estar vinculados al riesgo de ATBIO, siendo los sujetos muy susceptibles al aburrimiento quienes tienen menos probabilidades de informar tales accidentes (19). Adicionalmente, la evidencia actual es limitada para determinar la influencia de las características de la personalidad sobre la exposición accidental ocupacional de riesgo biológico a nivel nacional y local. En la literatura existe solo un reporte que sugiere una asociación entre los rasgos de personalidad del personal de enfermería y la ocurrencia de accidentes laborales de riesgo biológico (19).

Diferentes instrumentos de evaluación de personalidad han sido diseñados y validados; sin embargo, el 16PF es uno de los más utilizados desde hace más de 50 años, ha mejorado sensiblemente su validez y fiabilidad en su versión más reciente (16PF-5). Este cuestionario se basa en procedimientos empíricos de análisis factorial de los 16 rasgos de personalidad de primer orden (20, 21), y es considerado la construcción psicométrica en término de polaridades bajas y altas. Así mismo, el 16PF considera las dimensiones globales y las escalas sobre estilos de respuesta (21). Este cuestionario resulta útil en el análisis de rasgos de personalidad asociados a patrones de comportamiento volitivos determinantes en el ámbito profesional (21). Aunque tiene algunas desventajas tales como, no determinar qué ítems forman parte de cada uno de los rasgos de primer orden, ni cómo estos se combinan para formar las cinco dimensiones globales de personalidad. Otra desventaja puede ser la calidad de la traducción y la "comparabilidad métrica" en la adaptación de pruebas y cuestionarios, por lo tanto, es necesario garantizar que las mediciones obtenidas sean comparables entre las diferentes versiones como ha sido previamente publicado (22-24).

De acuerdo a los datos conocidos, este es el primer estudio de casos y controles que investiga si existe una asociación entre los rasgos de personalidad del personal de enfermería y la ocurrencia de accidentes de trabajo de riesgo biológico. El objetivo de este estudio fue establecer la asociación entre el rasgo de personalidad caracterizado por despreocupación y la ocurrencia de accidentes de trabajo de riesgo biológico en el personal de enfermería del Hospital Universitario de Santander (HUS) entre el 2008 y 2009. Para este propósito se utilizó el cuestionario $16 \mathrm{PF}$.

\section{Metodología}

Este estudio fue aprobado por el Comité de Ética para la Investigación Científica de la Facultad de Salud de la Universidad Industrial de Santander en el año 2011. Este proyecto se clasifica como de "riesgo mínimo", de acuerdo con lo expresado en el numeral B del Artículo 10 de la resolución 8430 de octubre de 1993, debido a que no implicó procedimientos invasivos físicos ni emocionales que puedan afectar a los participantes.

Se realizó un estudio observacional-analítico tipo casos y controles (25), con el objetivo de establecer la asociación despreocupación como rasgo de personalidad y la ocurrencia de ATBIO en el personal de enfermería del HUS durante los años 2008 y 2009. Se consideró caso a quienes presentaron y reportaron un ATBIO durante el año 2008 y 2009; y como control, a quienes no presentaron un ATBIO.

Los criterios de inclusión para el estudio fueron: ser trabajador de enfermería o auxiliar de enfermería que hubiese realizado sus actividades asistenciales durante el año 2008 y 2009. Además, que se encontraran laborando en el HUS al momento de la recolección de la muestra. Se consideró que para ser incluido en el estudio debía ser mayor de 18 años y menor de 65 años. La participación en el estudio fue voluntaria en quienes aceptaron el consentimiento informado.

En total los sujetos de estudio fueron 68 casos y 290 controles seleccionados por muestreo aleatorio simple. El tamaño de la muestra se calculó según previos estudios (25) tomando en consideración la prevalencia de exposición en los controles. Se escogió el rasgo de personalidad despreocupación en una muestra de 30 trabajadores de la salud (enfermeras, médicos, auxiliares de enfermería), la cual fue del $33.33 \%$ en el personal de enfermería del HUS. El poder deseado fue del $80 \%$, el nivel de significancia estadística fue un alfa de 0.05 y Odds Ratio (OR) de 2.5. El muestreo fue no probabilístico para los casos, por cuanto se seleccionaron el $92 \%$ de los accidentados, y probabilístico para la selección de controles, a través de muestreo aleatorio simple.

Los rasgos de personalidad fueron medidos con el inventario de 16 factores de personalidad (16 PF) de acuerdo con su manual (20), el cual comprende 187 ítems construidos para medir las 16 escalas primarias y cuatro dimensiones globales 
de la personalidad, incluye la evaluación del grado en que las personas pretenden mostrar una imagen distorsionada de sí misma. Cada ítem indaga sobre comportamientos comunes de las personas y se solicita al sujeto que evalúe de acuerdo a la situación más precisa, que lo describa entre tres opciones de respuestas tipo Likert (20). Los coeficientes de consistencia interna para cada factor se encuentran entre 0.35 y 0.83 (26).

Las variables para control de sesgos fueron recolectados por medio de una encuesta estructurada, incluyendo edad, sexo, cargo, tiempo de experiencia en el cargo, antigüedad en la empresa, ocupación adicional, horas de trabajo laboradas a la semana, horas adicionales trabajadas a la semana, disponibilidad de guantes cuando los requiere, práctica de reencapsulamiento, percepción de la probabilidad de presentarse un accidente, número de pacientes asignados, capacitación recibida en prevención de ATBIO, conocimiento del procedimiento a seguir en caso de ATBIO.

El investigador principal realizó el diligenciamiento de las encuestas. Se verificó que todos los datos generados se registraran en los formatos previamente diseñados y estandarizados para tal fin. Posterior a la verificación de los instrumentos de recolección de información, a cada uno de los sujetos se les asignó un código interno con el cual se identificó durante todo el estudio; esto con el objetivo de garantizar la confidencialidad en los datos obtenidos. La información recolectada se transcribió en una base de datos doblemente digitado por dos personas; posteriormente, se sometió a comprobación con el subprograma VALIDATE del programa estadístico EPI-INFO 6.04d (27).

Para el análisis estadístico se utilizó el programa STATA/SE 10.1 (Statacorp. LP; USA) (28). Se consideraron diferencias significativas con una p menor de 0.05 . Se realizó el cálculo de proporciones e intervalos de confianza, la comparación de proporciones se realizó por la prueba de chi cuadrado de Pearson. Para evaluar los factores asociados al ATBIO se realizó regresión logística, utilizando la variable explicativa principal el rasgo despreocupación, y se ajustó con las variables con valor de p menor de 0.20 en el análisis bivariado (29). Al modelo final se le realizó evaluación de la presencia de error de especificación, bondad de ajuste del modelo, análisis de residuales de Pearson a partir de la prueba de Hosmer-Lemeshow y los residuales deviance. Se calculó el cambio en el chi cuadrado (Delta chi2), el cambio en el deviance (Delta deviance) y evaluación de la discriminación del modelo (29).

\section{Resultados}

Se incluyeron $68(18 \%)$ casos y 290 controles. La prevalencia de ATBIO en la población estudiada fue de $10.7 \%$ en el 2008 y de $11.4 \%$ en el 2009 (Figura suplementaria 1). La edad de los sujetos está en el rango de 20 y 56 años, con un promedio de 36.3 años (desviación estándar (DE) 10 años), una mediana de 36 años y un rango intercuartílico de 18 años (Tabla 1).

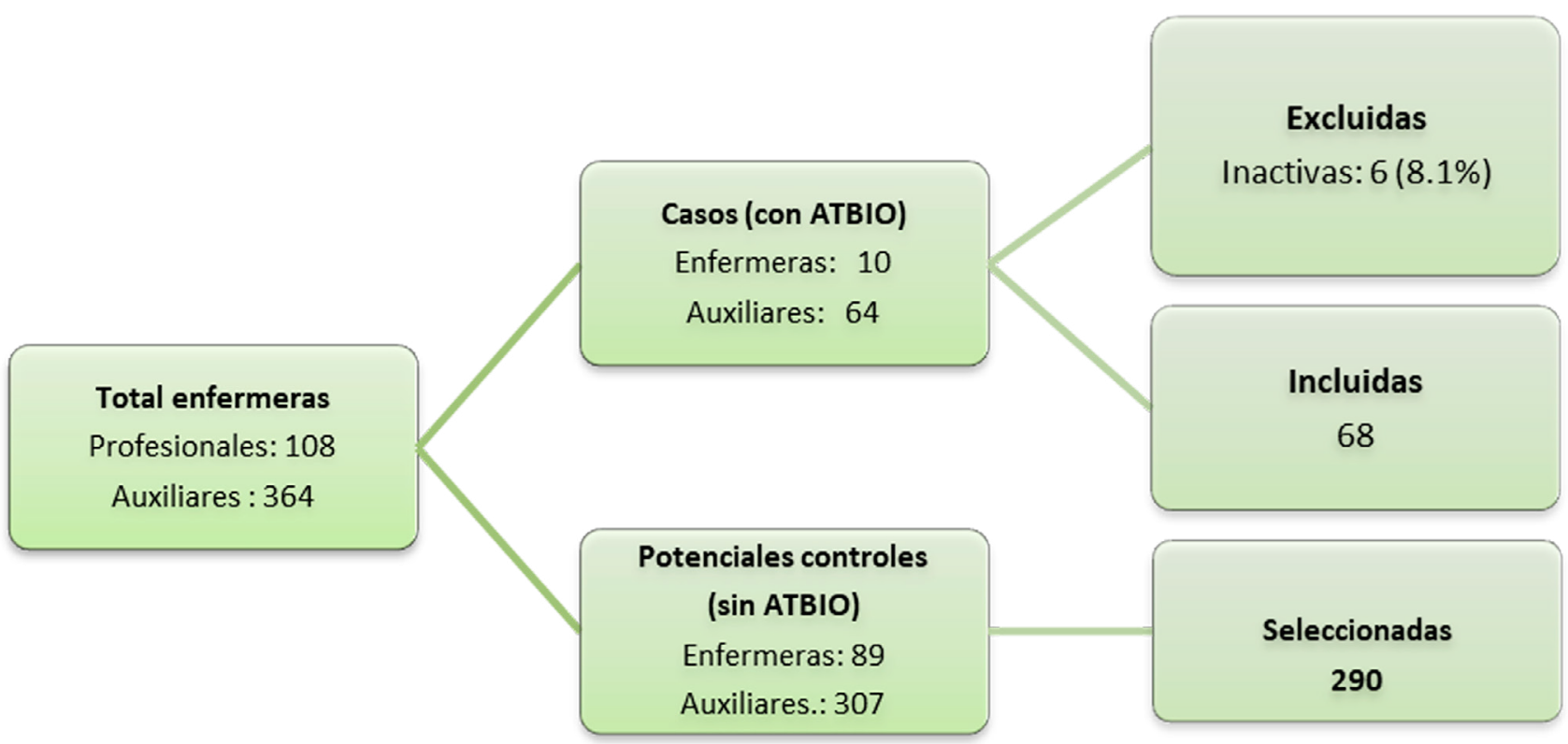

Figura suplementaria 1. Flujograma de inclusión de los participantes en el estudio

Fuente: elaboración propia. 
Tabla 1. Descripción de variables demográficas.

\begin{tabular}{|c|c|c|c|c|c|c|c|}
\hline \multirow{2}{*}{\multicolumn{2}{|c|}{ Variable }} & \multicolumn{3}{|c|}{ Global } & \multirow{3}{*}{$\begin{array}{r}\text { Caso }(n=68) \\
60(88.24 \%)\end{array}$} & \multirow{2}{*}{$\begin{array}{l}\text { Control } \\
(n=290)\end{array}$} & \multirow{2}{*}{$p$} \\
\hline & & n & $\%$ & IC 95\% & & & \\
\hline \multirow{2}{*}{ Sexo } & mujer & 316 & 88.2 & $84.4-91.4$ & & $256(88.28 \%)$ & \multirow{2}{*}{0.93} \\
\hline & hombre & 42 & 11.7 & $83-15$ & $8(11.7 \%)$ & $34(11.7 \%)$ & \\
\hline \multirow{3}{*}{ Edad } & $>=35$ años & 180 & 50.3 & $44-55$ & $28(15.5 \%)$ & $152(84.4 \%)$ & \multirow{3}{*}{0.01} \\
\hline & $27-34$ & 109 & 30.4 & $25-35$ & $18(16.51 \%)$ & $91(83.4 \%)$ & \\
\hline & < 27 años & 69 & 19.3 & $15-23$ & $22(31.8 \%)$ & $47(68.1 \%)$ & \\
\hline
\end{tabular}

Fuente: elaboración propia.

En la descripción de la población por grupos, solo la variable edad tuvo una distribución significativamente diferente en los casos y en los controles $(p=0.029)$. En la distribución por cargo, tiempo de permanencia del personal en el HUS, y la distribución por servicios no se reportó ninguna diferencia entre los casos y los controles.
La variable experiencia menor o igual a 4 años no presentó diferencias significativas entre los grupos $(\mathrm{p}<$ 0.20). El rango del tiempo de permanencia estuvo entre uno y cinco años, un promedio de 3.6 años (DE: 1.6 años) y una mediana de 5 años, con un rango intercuartílico de 3 (Tabla 2).

Tabla 2. Descripción de factores laborales

\begin{tabular}{|c|c|c|c|c|c|c|c|}
\hline \multicolumn{2}{|c|}{ Variable } & $\mathbf{n}$ & $\%$ & IC $95 \%$ & caso $(n=68)$ & control $(n=290)$ & $\mathbf{p}$ \\
\hline \multirow{2}{*}{ Caro } & Auxiliares & 270 & 75.42 & $70.6-79.7$ & $51(75 \%)$ & $219(75.52 \%)$ & 0.92 \\
\hline & Profesionales & 88 & 24.58 & $20.2-29.3$ & $17(25 \%)$ & $71(24.48 \%)$ & \\
\hline \multirow{2}{*}{ Experiencia } & $\iota=1$ año & 33 & 9.2 & $6-12$ & $11(16.1 \%)$ & $22(7.5 \%)$ & 0.02 \\
\hline & $>=1$ año & 325 & 90.7 & $87-93$ & $57(83.8 \%)$ & $268(92.4 \%)$ & \\
\hline \multirow{2}{*}{ Años en el HUS } & $\iota=2$ año & 95 & 26.5 & $22-31$ & $22(23.1 \%)$ & $73(76.84 \%)$ & 0.22 \\
\hline & $>=2$ año & 263 & 73.4 & $68-77$ & $46(17.4 \%)$ & $217(82.51 \%)$ & \\
\hline \multirow{2}{*}{$\begin{array}{l}\text { Labora en otra } \\
\text { institución de salud }\end{array}$} & si & 75 & 20.95 & $16.8-25.5$ & $17(25 \%)$ & $58(20 \%)$ & 0.36 \\
\hline & no & 283 & 79.05 & $74.4-83.1$ & $51(75 \%)$ & $232(80 \%)$ & \\
\hline \multirow{4}{*}{ Servicio } & hospitalización & 163 & 45.5 & $40-50$ & $24(35.2 \%)$ & $139(47.9 \%)$ & \\
\hline & Urgencias & 64 & 17.8 & $14-22$ & $18(26.4 \%)$ & $46(15.8 \%)$ & 0.11 \\
\hline & C extrema & 15 & 4.1 & $2.3-6.7$ & $2(2.9 \%)$ & $13(4.4 \%)$ & \\
\hline & 3 piso & 116 & 32.4 & $27.5-37.5$ & $24(35.2 \%)$ & $92(33.1 \%)$ & \\
\hline \multirow{2}{*}{ Numero de pacientes } & $>10$ & 118 & 32.9 & $28-38$ & $22(32.3 \%)$ & $96(33.1 \%)$ & 0.9 \\
\hline & $\iota=10$ & 240 & 67 & $61-71$ & $46(67.6 \%)$ & $194(66.9 \%)$ & \\
\hline \multirow{2}{*}{$\begin{array}{l}\text { Horas laboradas a la } \\
\text { semana dentro del HUS }\end{array}$} & $>=48$ & 172 & 48 & $42-53$ & $36(52.9 \%)$ & $136(46.9 \%)$ & 0.36 \\
\hline & $\iota=48$ & 186 & 51.9 & $46-57$ & $32(47 \%)$ & $154(53.1 \%)$ & \\
\hline \multirow{2}{*}{$\begin{array}{l}\text { Horas laboradas a las } \\
\text { semana fuera del HUS }\end{array}$} & $>=24$ & 58 & 16.2 & $12-20$ & $17(25 \%)$ & $41(14.1 \%)$ & 0.02 \\
\hline & $\iota=24$ & 300 & 83.8 & $79-87$ & $51(75 \%)$ & $249(85.5 \%)$ & \\
\hline \multirow{2}{*}{$\begin{array}{l}\text { Horas Totales laboradas } \\
\text { a la semana }\end{array}$} & $>=52$ & 84 & 23.4 & $19-28$ & $21(25 \%)$ & $63(75 \%)$ & 0.1 \\
\hline & $\iota=52$ & 274 & 76.5 & $71-80$ & $47(17.15 \%)$ & $227(82.8 \%)$ & \\
\hline
\end{tabular}

Fuente: elaboración propia.

En relación con variables relacionadas a la carga laboral (número de pacientes y horas laboradas) no se encontró diferencias significativas entre los dos grupos, excepto la variable laborar 24 horas o más adicionales a la semana $(p<0.02)$, reflejando diferencias significativas entre los casos y los controles. El 48\% del personal de enfermería trabaja más de 48 horas dentro del HUS, y el 23\% del personal trabaja en total más de 52 horas a la semana, sin encontrarse diferencias en estas dos variables entre los dos grupos (Tabla 2). 
En cuanto a prácticas y condiciones laborales, las variables disponer de guantes cuando se requieren y percibir el riesgo como alto, mostró diferencias significativas entre los grupos ( $\mathrm{p}=0.04 \mathrm{y} \mathrm{p}=0.02$, respectivamente). La variable reencapsular las agujas en su práctica laboral mostró una diferencia significativa entre casos y controles $(\mathrm{p}<0.20)$, las demás variables no mostraron cambios entre el grupo de casos y controles. En las variables relacionadas con rasgos de personalidad no hubo diferencias significativas entre los casos y los controles.

En cuanto al análisis bivariado, la variable edad menor o igual a 27 años evidenció asociación positiva (OR: 2.5; valor de $\mathrm{p}<0.05$ ); mientras que las variables cargo y sexo no mostraron asociación. La variable percibir la probabilidad de presentarse un accidente de trabajo de riesgo biológico como alto, mostró una asociación protectora y estadísticamente significativa para el ATBIO (OR: 2.5; valor de $\mathrm{p}<0.05$ ).

El análisis de factores laborales determinó que las siguientes variables tienen una asociación protectora, en orden de mayor a menor OR: trabajar adicionalmente por fuera del HUS en un tiempo igual o más de 24 horas a la semana (OR: 2; IC 95\%: 1.06-3.84), laborar en el servicio de urgencias (OR: 1.9; IC 95\%: 1.02 - 3.64), disponer de guantes siempre que se requieren (OR: 1.8; IC 95\% 1.01 -3.28), y la experiencia menor o igual a 4 años (OR:1.71; IC 95\% $0.92-3.28$ ) (Tabla 3).

Tabla 3. Asociación con factores laborales

\begin{tabular}{|c|c|c|c|c|}
\hline \multicolumn{2}{|c|}{ Variable } & OR & IC $95 \%$ & $\mathbf{P}$ \\
\hline \multirow{2}{*}{ Laborar en otra institución de salud } & No & ref. & $0.71-2.4$ & 0.36 \\
\hline & Sí & 1.34 & $0.71-2.5$ & 0.37 \\
\hline \multirow{2}{*}{ Reencapsular } & nunca & ref. & & \\
\hline & casi siempre y siempre & 1.44 & $0.88-2.48$ & 0.18 \\
\hline \multirow{2}{*}{$\begin{array}{c}\text { Disposición de guantes siempre } \\
\text { que se requiere }\end{array}$} & Sí & ref. & & \\
\hline & No & 1.82 & $1.01-3.289$ & 0.045 \\
\hline \multirow{2}{*}{ Percepción riesgo } & nulo - medio & ref. & & \\
\hline & alto & 0.54 & $0.31-0.92$ & 0.024 \\
\hline \multirow{2}{*}{$\begin{array}{l}\text { Haber recibido capacitación en } \\
\text { prevención ATBIO }\end{array}$} & Sí & ref. & & \\
\hline & No & 1.24 & $0.41-3.7$ & 0.69 \\
\hline \multirow{2}{*}{$\begin{array}{l}\text { Reporta que conoce el procedi- } \\
\text { miento }\end{array}$} & Sí & ref. & & \\
\hline & No & 1.37 & $0.55-3.41$ & 0.49 \\
\hline \multirow{2}{*}{$\begin{array}{l}\text { concepto de conocimiento de pro- } \\
\text { cedimiento ATBIO }\end{array}$} & Sí & ref. & & \\
\hline & No & 1.27 & $0.73-2.19$ & 0.39 \\
\hline \multirow{5}{*}{ Servicio } & otros & ref. & & \\
\hline & C externa & 0.63 & $0.14-2.93$ & 0.57 \\
\hline & Urgencias & 1.9 & $1.02-3.64$ & 0.04 \\
\hline & 3-piso (UCI, cirugía y partos) & 1.69 & $0.81-2.84$ & 0.18 \\
\hline & hospitalización & 0.59 & $0.34-1.00$ & 0.061 \\
\hline \multirow{2}{*}{ Número de pacientes } & $<10$ & ref. & & \\
\hline & $>10$ & 0.96 & $0.52-1.75$ & 0.9 \\
\hline \multirow{2}{*}{ Años en el HUS } & $>2$ & ref. & & \\
\hline & $<=2$ & 1.42 & $0.75-2.59$ & 0.22 \\
\hline \multirow{2}{*}{ Experiencia en años } & $>1$ & ref. & & \\
\hline & $<=1$ & 2.35 & $0.96-5.38$ & 0.02 \\
\hline \multirow{2}{*}{$\begin{array}{c}\text { Horas laboradas a la semana dentro } \\
\text { del HUS }\end{array}$} & $<48$ & ref. & & \\
\hline & $>=48$ & 1.27 & $0.72-2.24$ & 0.36 \\
\hline \multirow{2}{*}{$\begin{array}{l}\text { Horas laboradas adicionales a la } \\
\text { sema }\end{array}$} & $<24$ & ref. & & \\
\hline & $>=24$ & 2.02 & $1.06-3.84$ & 0.03 \\
\hline \multirow{2}{*}{ Horas totales laboradas a la semana } & $<52$ & ref. & & \\
\hline & $>=52$ & 1.6 & $084-2.98$ & 0.12 \\
\hline
\end{tabular}

Fuente: elaboración propia. 
Se analizaron los OR en cada uno de los rasgos por calificación en la escala como polo bajo (calificaciones entre 1-3), polo neutro (calificaciones 4-7) y polo alto (calificaciones entre 8-10), de acuerdo con el manual (20). Se utilizó como referencia el valor medio por cuanto el rasgo es neutro (calificaciones 4-7). No se encontró asociación significativa entre el ATBIO y el rasgo G despreocupación. Sin embargo, se encontraron rasgos de personalidad factor E polo bajo-Débil, factor M polo alto-Imaginativo, factor Q1 polo alto-crítico fueron estadísticamente significativos $(p<0.20)$. Para evaluar la confusión se realizó análisis estratificado de MantelHaenzel (M-H), y dado que la variable explicativa principal factor G. Despreocupado-Escrupuloso en su polo alto no mostró asociación significativa con el accidente de trabajo de riesgo biológico, se le realizó análisis estratificado por las demás variables, con $\mathrm{p}<0.20$ consideradas como potencialmente confusoras en esta relación, de lo cual no se generaron diferencias entre los OR crudo y el OR ajustado, y el test de homogeneidad no fue significativo.

Como parte del modelo final se realizó siguiendo un orden jerárquico paso a paso, de allí se incluyeron las variables con $\mathrm{p}<0.05$ y que en la prueba de Log-Likelihood se fundamentara si debía ser incluida o no en el modelo, teniendo en cuenta la forma funcional de la variable (Tabla 4). De lo anterior se determinó el siguiente modelo final explicativo con la variable dependiente principal factor GDespreocupación. (Tabla 5).

Tabla 4. Variables candidatas a incluir en el modelo multivariado

\begin{tabular}{lccc}
\multicolumn{1}{c}{ Variable } & OR crudo & IC 95\% & $P$ \\
Edad en años $\leq 27$ & 1.86 & $1.01-3.37$ & 0.020 \\
Experiencia $\leq 4$ años & 1.71 & $0.92-3.11$ & 0.059 \\
\hline Horas laborales adicionales a la semana (ref. $<24 \mathrm{~h})$ & 2.02 & $1.06-3.84$ & 0.031 \\
\hline Persepción riesgo (ref. nulo, medio) & 0.54 & $0.31-0.92$ & 0.024 \\
\hline Horas totales laborales a la semana (ref. $<52$ ) & 1.60 & $0.84-2.98$ & 0.111 \\
\hline Servicio de Urgencias & 1.9 & $1.02-3.64$ & 0.042 \\
Reencapsular & 1.44 & $0.88-2.48$ & 0.181 \\
\hline Disposición de guantes siempre que se requiere & 1.82 & $1.01-3.28$ & 0.045 \\
Servicio Hospitalización & 0.59 & $0.34-1.00$ & 0.061 \\
Rasgo E bajo (Débil-Dominante) & 1.49 & $0.82-2.41$ & 0.201 \\
\hline Rasgo Q1 alto (Conservador-Crítico) & 2.49 & $0.79-7.79$ & 0.116 \\
\hline Rasgo M2 alto (Convencional-Imaginativo) & 2.39 & $0.91-6.28$ & 0.076
\end{tabular}

Fuente: elaboración propia.

Tabla 5. Modelo final con la variable independiente principal despreocupación

\begin{tabular}{lccc}
\multicolumn{1}{c}{ ATBIO } & Odds Ratio & $\boldsymbol{P}$ & IC 95\% \\
Despreocupación & 0.88 & 0.730 & $0.44-1.76$ \\
Percibir el riesgo como alto & 0.42 & 0.004 & $0.23-0.75$ \\
Trabajar $\geq$ 24 horas adicionales a la semana & 2.66 & 0.005 & $1.34-5.28$ \\
\hline Servicio Urgencias & 2.77 & 0.004 & $1.38-5.35$ \\
No disponer de guantes cuando se requiere & 2.05 & 0.022 & $1.10-3.79$
\end{tabular}

Fuente: elaboración propia.

El modelo final está correctamente especificado por cuanto la prueba indica que las variables explicatorias son suficientes para predecir el logit ya que arrojó un valor de $\mathrm{p}<0.05$ (15). Así mismo, indica que no hay evidencia de error de especificación por omisión de variables importantes por cuanto el valor de $\mathrm{p}$ del cuadrado de la estimación no es significativo $(\mathrm{p}=0.59)$.
La evaluación de la bondad de ajuste del modelo, a través de la prueba lfit de Stata/SE10.0 (Stata Corp. LP; USA) (28), muestra un valor de $p=0.26$, el cual es no significativo, lo que sugiere que hay un buen ajuste.

De la prueba de Hosmer y Lemeshow (1989) se obtuvo un valor de $\mathrm{p}$ no significativo, lo cual indica que una $\mathrm{p}$ 
de 0.33 apoya la hipótesis nula y sugiere que el modelo propuesto continúa ajustando razonablemente los datos. El área bajo la curva es de 0.72 , lo cual indica un aceptable poder discriminatorio del modelo, con un punto de corte de 0.5 el modelo clasifica correctamente el $80.73 \%$ de las observaciones (Figura 1). Las conclusiones del modelo final no se ven afectadas al excluir los patrones outlier e influyentes, la dirección de las asociaciones no cambia, y se mantiene estadísticamente significativa, los límites de los intervalos de confianza no presentan mayores cambios (Tabla 6).

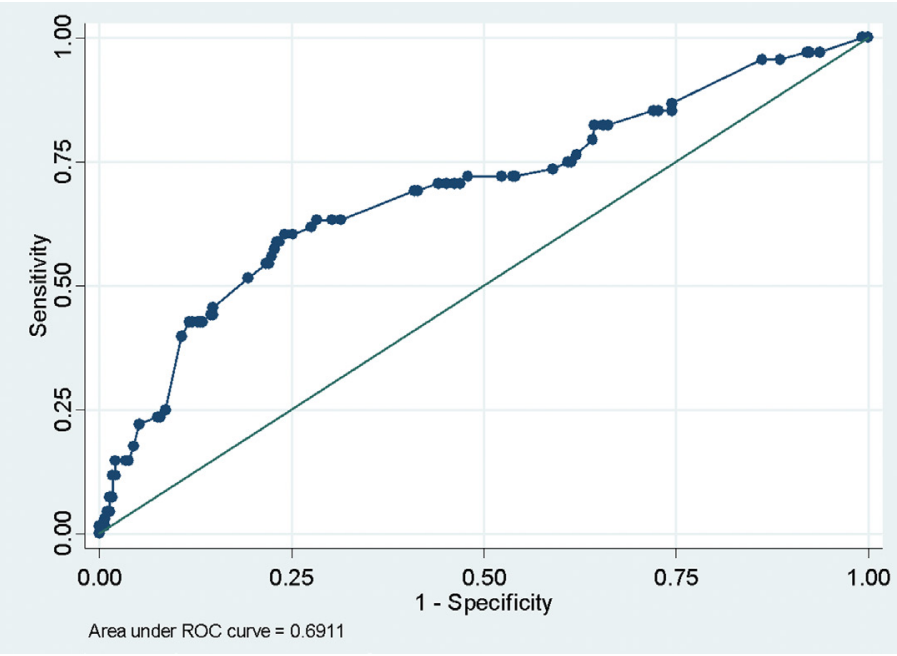

Figura 1. Curva receptor operador (ROC) del modelo logístico final

Fuente: elaboración propia.

Tabla 6. Modelo final sin outliers ni patrones influyentes

\begin{tabular}{|c|c|c|c|c|c|c|}
\hline \multirow[t]{2}{*}{ Variables modelo } & \multicolumn{2}{|c|}{$\begin{array}{c}\text { Modelo Final } \\
\text { n: } 358\end{array}$} & \multicolumn{2}{|c|}{$\begin{array}{c}\text { Sin Outliers } \\
\text { n: } 358\end{array}$} & \multicolumn{2}{|c|}{$\begin{array}{l}\text { Sin Outliers, Sin } \\
\text { Influyentes } \\
\text { n: } 345\end{array}$} \\
\hline & OR & $\mathbf{p}$ & OR & p & OR & p \\
\hline Servicio Urgencias vs. Otros & 2.8 & 0.002 & 2.8 & 0.002 & 3.2 & 0.001 \\
\hline Percibir el riesgo como alto & 0.42 & 0.004 & 0.42 & 0.004 & 0.38 & 0.002 \\
\hline Horas fuera $\geq 24$ horas a la semana & 2.6 & 0.005 & 2.6 & 0.005 & 2.1 & 0.047 \\
\hline $\begin{array}{l}\text { No Disponer de guantes siempre que se } \\
\text { requiere }\end{array}$ & 1.95 & 0.034 & 1.95 & 0.034 & 2.4 & 0.001 \\
\hline experiencia $\leq 1$ año & 2.31 & 0.041 & 2.31 & 0.04 & 2.53 & 0.026 \\
\hline
\end{tabular}

Fuente: elaboración propia.

En resumen, se determinó que el personal que labora en el servicio de urgencias tiene tres veces más riesgo de presentar un ATBIO, comparado con otras enfermeras y auxiliares de enfermería de características socio demográficas y situacionales similares. Así mismo, un sujeto que perciba la no disposición de guantes cuando se requieren durante su labor tiene dos veces más riesgo de presentar un ATBIO. Los trabajadores de enfermería que laboran fuera del HUS más de 24 horas tienen 2.6 veces más riesgo de presentar un ATBIO frente a aquellos sujetos que no lo hacen. De igual manera, el personal de enfermería que percibe mayor riesgo de presentar un ATBIO se relaciona con la disminución del riesgo en el 60\% (OR: 0.40).

\section{Discusión}

Uno de los principales objetivos del sistema General de Riesgos Profesionales en Colombia es la prevención de riesgos ocupacionales, incluyendo AT y enfermedad profesional (1). Existe evidencia suficiente que sustenta 
que los ATBIO de mayores frecuencias antes de diciembre 2019 era la infección por los virus de Hepatitis B, Hepatitis C y VIH; por lo cual, la identificación de las causas que generan dicha accidentalidad permitirá establecer estrategias que controlen y disminuyan la siniestralidad laboral y el control de costos directos e indirectos derivados de su ocurrencia de ATBIO.

En el presente estudio se investigó la asociación entre el rasgo de personalidad y la ocurrencia de accidentes de trabajo de riesgo biológico en el personal de enfermería del Hospital Universitario de Santander (HUS) entre el 2008 y 2009, utilizando el cuestionario 16 PF. Los resultados reportados concuerdan con las variables incluidas en estudios previos referente a la accidentalidad laboral de riesgo biológico $(30,31)$, entre estas, trabajar más de 24 horas a la semana fuera del HUS, no disponer de guantes cuando se requieren, laborar en el servicio de urgencias y percibir como alta la probabilidad de presentarse un accidente de trabajo de riesgo biológico.

La variable horas de trabajo adicional fuera del HUS mayores o iguales a 24 horas concuerda con los hallazgos de un estudio de casos y controles realizado en Brasil, el cual incluyó 787 enfermeras. Dicho estudio reportó una asociación significativa y un OR 2.47, tomando la variable como horas a la semana $>50$. Otro estudio de corte trasversal realizado en Turquía con 449 enfermeras encontró una asociación protectora con actividad laboral de más de 8 horas al día con un OR de 1.91 (IC95\% 1.073.38) (33). Para efectos del estudio, se encontró que el $20 \%$ del personal trabaja horas adicionales fuera del HUS. Esto implica que tanto la exposición como acciones de prevención no son factibles al ser implementadas por el HUS. Actualmente no existe en la legislación colombiana, ni en las normas técnicas de salud ocupacional, una restricción específica en relación con exposición a riesgos biológicos en más de un lugar de trabajo. En el trascurso de la recolección de datos, varios participantes expresaron la necesidad de complementar sus ingresos laborales realizando jornadas de trabajo adicionales, debido a la contratación actual y para el cubrimiento de las necesidades básicas.

El desarrollar funciones dentro del servicio de urgencias presentó un mayor riesgo de ocurrencia de ATBIO, lo cual no había sido previamente reportado. En la gran mayoría de estudios se tiene como referencia el área quirúrgica. Smith y colaboradores (31), encontraron que laborar en la unidad materno- neonatal fue un factor protector (OR: 0.3; IC 95\%: $0.1-0.7$ ); mientras que trabajar en el área quirúrgica es factor de riesgo comparado con el área clínica (OR: 1.75; IC 95\%: 1.12-2.75) (31), similar al riesgo reportado por otro estudio (OR: 1.76; IC95\% 1.13 -
2.79)(34). Otra investigación encontró que trabajar en los servicios de psiquiatría, pediatría y neonatos, son factores protectores (30). En el presente estudio, al comparar el área quirúrgica (quirófano) con los otros servicios, no se reportó ninguna asociación (OR:1.39; IC95\% 0.48 - 3.58; $\mathrm{p}=0.46$ ). Por lo anterior, se podría presumir que el servicio de urgencias del HUS tiene ciertas características que lo hacen estar relacionado positivamente con la ocurrencia de ATBIO en el personal de enfermería, los cuales deben ser estudiados.

En el presente estudio se encontró que en el servicio de urgencias el personal de enfermería labora 42 horas a la semana, lo cual es un tiempo menor comparado con los otros servicios que laboran hasta 48 horas a la semana. La variable horas a la semana laboradas en el HUS no generó asociación significativa; así como tampoco el número de pacientes asignados al cuidado, por lo tanto, es necesario identificar otras variables como el estrés laboral, las actividades y procedimientos que allí se realizan, la atención a familiares, el espacio físico, condiciones laborales y disponibilidad de recursos físicos y humanos, lo cual podría suministrar mayor evidencia para determinar esta relación y establecer programas focalizados de prevención de accidentes de trabajo en esta área.

La percepción del riesgo en este estudio fue investigada como la autoevaluación de la posibilidad de que se presente un ATBIO en el servicio en el cual cada sujeto se encuentra laborando. Anteriormente, otro estudio incluyó percepción del riesgo, el cual determinó que en los sujetos que percibían el riesgo como bajo el OR fue de 10.19 (IC 95\%: 3.64-28.32) y quienes lo perciben como medio se encontró un OR de 4.88 (2.69-8.79) en el servicio de enfermería de un hospital brasilero. Además, se halló una asociación protectora para aquellos sujetos que autoreportaron la probabilidad de accidentarse como alta con un OR 0.40 (IC95\% 0.23-0.75). La percepción del riesgo es susceptible de control en la población de enfermería, a través de programas de educación continua y la utilización de metodologías que incluyen situaciones aprendidas y socialización de consecuencias y riesgos relacionados con el ATBIO, pues se logró determinar que esta variable podría disminuir en un $60 \%$ el riesgo de sufrir un ATBIO en el personal expuesto.

La investigación también evaluó la percepción por parte del personal de enfermería de la disponibilidad de guantes cuando se requieren, puesto que posiblemente genera mayor seguridad y confianza en la realización de un procedimiento. El estudio logró identificar que este aspecto genera una mayor probabilidad de presentarse un ATBIO (OR: 1.95; IC95\% 1.05-3.64). Al mismo tiempo, cuando la aguja perfora el guante, el contaminante potencial 
disminuye su capacidad de patogenicidad, motivo por lo cual se considera de vital importancia garantizar por parte del empleador la disponibilidad de los guantes como elementos de protección personal para la realización de actividades que así lo requieran.

Para finalizar, este estudio no logró demostrar la posible asociación existente entre los rasgos de personalidad y la ocurrencia del ATBIO en el personal de enfermería del HUS por varias posibles razones. En primer lugar, al analizar el poder para establecer la asociación de la hipótesis, se calculó una proporción del rasgo despreocupación en los controles del $33 \%$, según registro de exámenes de ingreso en otra población de salud. En la muestra obtenida, este rasgo está en el $20.7 \%$ de los controles, cifra inferior al calculado. Sin embargo, al recalcular el tamaño de la muestra, teniendo en cuenta la exposición en los controles del $20.7 \%$ y para alcanzar el poder deseado del $80 \%$ y una confiabilidad del $95 \%$, un OR 2.5; el tamaño de la muestra que se obtuvo con este procedimiento está acorde con la muestra real en la cual se incluyó el 20\% adicional, calculando por pérdidas potenciales, las cuales fueron $8 \%$.

Otro aspecto a mencionar es el sesgo de selección, por cuanto los estudios de casos y controles se constituyen en los diseños en los cuales las probabilidades de muestreo de los diferentes grupos de efecto-exposición pueden resultar diferenciales (25), en el presente estudio el reporte del ATBIO (efecto) pudo hacerse o no dependiendo de los rasgos de personalidad del sujeto involucrado (exposición), por tanto, si algunos individuos reportaron el ATBIO y fueron incluidos en el grupo de casos debido a su personalidad, se puede presentar un odds de exposición sesgado en los casos, porque varía dependiendo de la exposición. Este hecho pudo distorsionar la verdadera asociación entre la exposición y el desenlace.

Aunque se realizaron campañas de sensibilización, búsqueda activa de casos, y se incluyó una pregunta de filtro para identificar sobre la práctica de reportar el accidente de trabajo, se encontró que solo una persona no había hecho el reporte de ATBIO. Esta pregunta está sujeta a que el individuo la responda positivamente por temor a un juzgamiento social, y más aún, conociendo la falta de reportes de los accidentes de trabajo implicaría sanciones económicas para la empresa, lo cual pudiera también repercutir sobre el trabajador. Además, se encontró que a pesar de que el $93 \%$ del personal de enfermería ha recibido capacitación en prevención de accidentes de trabajo, tan solo el $66 \%$ conoce el procedimiento que se debe seguir después de presentarse un ATBIO, es decir, que quienes desconocen el procedimiento pueden terminar por reportarlo contribuyendo así a diluir la asociación.
La forma de contratación del personal de enfermería, es decir, quienes son socios de una cooperativa de trabajo asociada, los tiempos e incapacidades deben ser asumidas por ellos mismos. Esto favorece a que en estos casos opten por la decisión de mantenerse laborando, y no decidan ausentarse de su trabajo a realizar los procesos requeridos de reporte de ATBIO, con el fin de no disminuir sus ingresos.

En segundo lugar, el sesgo de información dado por la definición imperfecta de las variables del estudio o por los procedimientos inadecuados para la recolección de datos con la prueba psicotécnica $16 \mathrm{PF}$. Esta prueba es ampliamente utilizada en el sector empresarial para la selección de personal, los cuales muestran pruebas de consistencia interna y reproducibilidad adecuadas (26). No obstante, las pruebas exploratorias realizadas al instrumento en el presente estudio, como el Alpha de Cronbach, reportaron muy baja consistencia interna; no se encontró evidencia de estudios de validez de criterio, los cuales comparan la prueba evaluada con el estándar de oro, lo cual sería un insumo mayor para calificar la prueba.

Es importante destacar que el 16 PF (20) genera el resultado de una variable que mide la distorsión en la cual incurre un sujeto al diligenciarla. Sin embargo, no se identificó que las respuestas al cuestionario estuviesen sujetas a tal distorsión o la manipulación del investigador, puesto que se midieron las mismas variables en diferentes preguntas. En este estudio no se encontraron diferencias significativas al incluir esta variable de control dentro del análisis de datos.

En cuanto a la definición imperfecta de la variable a estudio, que es otro factor que contribuye al sesgo de información, vale la pena mencionar que la construcción del concepto de personalidad se fundamenta en múltiples teorías, las cuales no han sido unificadas y su medición depende de la teoría en que se fundamente, y el 16PF no está acorde con una sola teoría.

\section{Conclusión}

Se identificaron variables significativas relacionadas con la ocurrencia de ATBIO descritas en resultados, las cuales son insumo para la planeación, ejecución e implementación de sistemas de gestión en seguridad y salud en el trabajo, dirigidos al control de la accidentalidad laboral de riesgo biológico. No fue posible identificar si los rasgos de personalidad influyen en la ocurrencia de ATBIO. 


\section{Declaración de conflictos de interés}

Los autores manifiestan que durante el desarrollo del trabajo no existieron conflictos de interés. El presente estudio fue financiado con recursos propios de los autores.

\section{Referencias}

1. Decreto 1295 de 1994 por el cual se determina la organización y administración del Sistema General de Riesgos Profesionales. Diario Oficial, número 41.405, (24 de junio de 1994).

2. Vergara Teresa, Véliz Elena, Fica Alberto, Dabanch Jeannette. Exposiciones a fluidos de riesgo en el personal desalud. Evaluación de gastos directos en sumanejo. Rev. chil. infectol. [Internet]. 2018: 490-497. Disponible en: https://www.scielo.cl/scielo.php?script=sci arttext\&pid $=$ S0716-10182018000500490

3. Arenas-Sánchez A, Pinzón-Amado A. Riesgo biológico en el personal de enfermería: una revisión práctica. Revista Cuidarte. 2011;2:216-24. doi: https:// doi.org/10.15649/cuidarte.v2i1.60

4. Montella E, Schiavone D, Apicella L, Di Silverio P, Gaudiosi M, Ambrosone E, et al. Cost-benefit evaluation of a preventive intervention on the biological risk in health: the accidental puncture during the administration of insulin in the University Hospital "Federico II" of Naples. Ann Ig. 2014;26(3):272-8. doi: https://doi.org/10.7416/ai.2014.1985

5. Rim KT, Lim CH. Biologically hazardous agents at work and efforts to protect workers' health: a review of recent reports. Saf Health Work. 2014 Jun;5(2):4352. doi: https://doi.org/10.1016/j.shaw.2014.03.006.

6. Mtengezo J, Lee H, Ngoma J, Kim S, Aronowitz T, DeMarco R, Shi L. Knowledge and Attitudes toward HIV, Hepatitis B Virus, and Hepatitis C Virus Infection among Health-care Workers in Malawi. Asia Pac J Oncol Nurs. 2016 Oct-Dec;3(4):344-351. doi: https://doi.org/10.4103/2347-5625.195921. PMID: 28083551 ; PMCID: PMC5214867.

7. Cekin AH, Cekin Y, Ozdemir A. The level of knowledge of, attitude toward and emphasis given to $\mathrm{HBV}$ and $\mathrm{HCV}$ infections among healthcare professionals: data from a tertiary hospital in Turkey. Int J Occup Med Environ Health. 2013 Mar;26(1):12231. doi: https://doi.org/10.2478/s13382-013-0077-3.

8. Joukar F, Mansour-Ghanaei F, Naghipour MR, Hasandokht T. Nurses' Knowledge toward Hepatitis B and Hepatitis C in Guilan, Iran. Open Nurs J. 2017 Apr 17;11:34-42. doi: https://doi.org/10.2174/187443460171 1010034. PMID: 28567168; PMCID: PMC5420166.

9. Liu Y, Ma C, Jia H, Xu E, Zhou Y, Zhang Z, Lu L, Rodewald L, Hao L. Knowledge, attitudes, and practices regarding hepatitis $\mathrm{B}$ vaccination among hospital-based doctors and nurses in China: Results of a multi-site survey. Vaccine. 2018 Apr 19;36(17):2307-2313. doi: https://doi.org/10.1016/j. vaccine.2018.03.018.

10. Huang Z, Zhao S, Li Z, Chen W, Zhao L, Deng L, Song B. La batalla contra la enfermedad del Coronavirus 2019 (COVID-19): Manejo de Emergencia y Control de Infecciones en un Departamento de Radiología. J Am Coll Radiol. 2020 Jul;17(7):e29-e36. doi: https:// doi.org/10.1016/j.jacr.2020.05.006.

11. Valdés PR, Cámera LA, Serna Mdl, Abuabara-Turbay Y, Carballo-Zárate V, Hernández-Ayazo $\mathrm{H}$, et al. Ataque al personal de la salud durante la pandemia de Covid-19 en Latinoamérica. Acta Med Col [Internet]. 31 de julio de 2020; 45(3). Disponible en: http://www. actamedicacolombiana.com/ojs/index.php/actamed/ article/view/1975

12. Martí-Amengual G, Sanz-Gallen P, ArimanyManso J. Valoración medicolegal de la infección por COVID-19 en el ámbito laboral. Rev Esp Med Legal. 2020;46:146-152. doi: http://doi.org/10.1016/j. remle.2020.05.008

13. Roberts BW. Back to the Future: Personality and Assessment and Personality Development. J Res Pers. 2009 Apr 1;43(2):137-145. doi: https://doi. org/10.1016/j.jrp.2008.12.015

14. Fajkowska M, Kreitler S. Status of the Trait Concept in Contemporary Personality Psychology: Are the Old Questions Still the Burning Questions? J Pers. 2018 Feb;86(1):5-11. doi: https://doi.org/10.1111/ jopy. 12335

15. Navarro JLM. Revista de psicología del trabajo y de las organizaciones. 1999;15:237-66. Disponible en: https://dialnet.unirioja.es/revista/1207/A/1999

16. Paula GI.Personalidad: un recorrido por los principales conceptos desarrolladossobre el constructo. Revista ConCiencia EPG. 2018;3(2):34-57. doi: https://doi. org/10.32654/CONCIENCIAEPG.3-2.3

17. Barrick MR, Mount MK, Li N. The Theory of Purposeful Work Behavior: The Role of Personality, Higher-Order Goals, and Job Characteristics. Academy of Management Review. 2013;38(1):132-53. Disponible en: https:// www.researchgate.net/publication/274693473 The Theory of Purposeful Work Behavior The Role of Personality_Higher-Order_Goals_and_Job Characteristics

18. Takase M, Yamamoto M, Sato Y. Effects of nurses' personality traits and their environmental characteristics on their workplace learning and nursing competence. Jpn J Nurs Sci. 2018 Apr;15(2):167-180. doi: https://doi.org/10.1111/jjns.12180.

19. Rabaud C, Zanea A, Mur JM, Blech MF, Dazy D, May T, Guillemin F. Occupational exposure to blood: 
search for a relation between personality and behavior. Infect Control Hosp Epidemiol. 2000 Sep;21(9):56474. doi: https://doi.org/10.1086/501805.

20. Cattell RB, Eber HW, Tatsuoka MM. Handbook for the sixteen personality factor questionnaire (16 PF) in clinical, educational, industrial, and research psychology, for use with all forms of the test. Institute for Personality and Ability Testing; 1970. 390. Disponible en: https://onesearch. library.rice.edu/permalink/01RICE INST/11atd6j/ alma991007673689705251

21. Aluja A, Blanch Á. Replicabilidad de los factores de segundo orden del 16PF-5 en muestras americanas y españolas. Psicothema. 2003;15(2):309-14. Disponible en: http://www.psicothema.com/ psicothema.asp?id $=1062$

22. Tamassia CV, Hambleton RK., Merenda PF, Spielberger, CD. Adapting educational and psychological tests for cross-cultural assessment. Psychometrika. 2007;72(4):649-51. doi: https://doi. org/10.1007/s11336-007-9014-3.

23. Balluerka N, Gorostiaga A, Alonso-Arbiol I, Haranburu M. La adaptación de instrumentos de medida de unas culturas a otras: Una perspectiva práctica. Psicothema. 2007;19(1):124-33. Disponible en: asp? id $=3338$

24. Saville P, Blinkhorn S. Reliability, homogeneity and the construct validity of Cattell's 16PF. Personality and Individual Differences. 1981;2(4):325-33. Disponible en: https://www.sciencedirect.com/science/article/ abs/pii/019188698190088X?via\%3Dihub

25. Breslow NE. Case-Control Studies. Handbook of Epidemiology. 2014: 293-232. https://doi. org/10.1007/978-0-387-09834-0 7

26. O'Dell J, Karson M, Karson $\overline{\mathrm{S}}$. 16PF-5 una guía para su interpretación en la práctica clínica: TEA Ediciones; 2011. 184.
27. Su Y, Yoon SS. Epi info - present and future. AMIA Annu Symp Proc. 2003;2003:1023. Disponible en: $\quad$ https://www.ncbi.nlm.nih.gov/pmc/articles/ PMC1480129/

28. Stata Statistical Software: Release 12. [Internet]. StataCorp LP. 2011. Disponible en: https://www.stata. com/stata12/

29. Suarez E, Perez CM, Rivera R, Martínez MN. Logistic Regression in Case-Control Studies. Applications of Regression Models in Epidemiology 2017: 165-26. doi: https://doi.org/10.1002/9781119212515.ch11

30. Clarke SP. Hospital work environments, nurse characteristics, and sharps injuries. Am J Infect Control. 2007 Jun;35(5):302-9. doi: https://doi. org/10.1016/j.ajic.2006.07.014.

31. Smith DR, Smyth W, Leggat PA, Wang RS. Needlestick and sharps injuries among nurses in a tropical Australian hospital. Int J Nurs Pract. 2006 Apr;12(2):71-7. Disponible en: https://onlinelibrary. wiley.com/doi/10.1111/j.1440-172X.2006.00553.X

32. Canini SR, Moraes SA, Gir E, Freitas IC. Percutaneous injuries correlates in the nursing team of a Brazilian tertiary-care university hospital. Rev Lat Am Enfermagem. 2008 Sep-Oct;16(5):818-23. doi: https://doi.org/10.1590/s0104-11692008000500004.

33. Ilhan MN, Durukan E, Aras E, Türkçüoğlu S, Aygün R. Long working hours increase the risk of sharp and needlestick injury in nurses: the need for new policy implication. J Adv Nurs. 2006 Dec;56(5):563-8. doi: https://doi.org/10.1111/j.1365-2648.2006.04041.x.

34. Nsubuga FM, Jaakkola MS. Needle stick injuries among nurses in sub-Saharan Africa. Trop Med Int Health. 2005 Aug;10(8):773-81. doi: https://doi. org/10.1111/j.1365-3156.2005.01453.x 